gen im Bereich „Public Affairs“ aber auch im Zusammenhang mit Aktivitäten von Corporate Social Responsibility stärker nachgefragt werden. Hier stehen rohstoffreiche Länder wie Peru (Silber), Chile (Kupfer), Bolivien (Erdgas, Wasser) oder Argentinien (Soja) im Vordergrund.

Interessant wird es sein, wie und ob lateinamerikanische Berater Besonderheiten und spezifische Fähigkeiten entwickeln, die sich aus den Bedingungen ihrer Märkte ergeben. So wie beispielsweise bereits in der Werbebranche brasilianische und argentinische Kreative dafür bekannt sind einen ganz eigenen Stil entwickelt zu haben, der bei Branchentreffen regelmäßig mit entsprechenden Auszeichnungen und Preisen bedacht wird, könnte ähnliches mit lateinamerikanischen Politik-Kommunikationsberatern geschehen. Herausforderungen gibt es im politischen Leben zwischen Rio Grande und Feuerland genug!

\section{Literatur}

Huntington, S. (1993). The Third Wave: Democratization in the Late Twentieth Century. University of Oklahoma Press.

Konrad-Adenauer-Stiftung. (2011). Demokratie-Index Lateinamerika 2011. IDD-LAT Anzurufen unter www.kas.de/wf/doc/ kas_28989-544-1-30.pdf?111005113120. [12.11.2012]

Weltbank (2012). Doing Business 2012. Abzurufen unter www.doingbusiness.org/rankings. [12.11.2012]

Transparency International (2011): Corruption Perceptions Index. Abzurufen unter http://cpi.transparency.org/cpi2011/.
Plasser F.; Plasser, G. (2002). La Campaña Global. Los nuevos Gurúes del Marketing Político en acción. Konrad Adenauer Stiftung (Hg.). Buenos Aires.

Layton, M. (2012). ¿Quién confía en los medios masivos de comunicación en América Latina? In: S. Mitchell, A. Smith, \& E. Zechmeister (Hg.). Perspectivas desde el Barómetro de las Américas: 2012 (74). Vanderbilt University. Abzurufen unter www.vanderbilt.edu/lapop/insights/ IO874_es.pdf. [12.11.2012]

Latinobarómetro (2012). Informes anuales. Abzurufen unter www.latinobarometro.org/latino/LATContenidos.jsp. [12.11.2012]

ComScore (2012). Latin America Leads as the Most Socially-Engaged Global Region with Users Spending 7.5 Hours on Social Networking Sites in April. (Pressemitteilung). Abzurufen unter http://www.comscore.com/ Insights/Press_Releases/2012/.[12.11.2012]

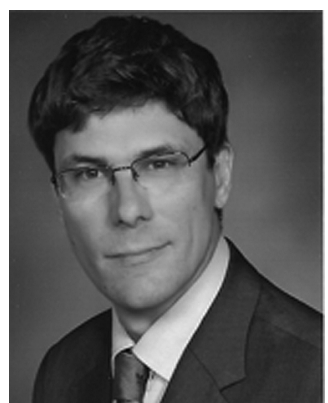

Peter-Alberto Behrens studierte Politikwissenschaften in Barcelona, Bonn und Mainz. Von 1998-2003 war er als Berater für die Hering Schuppener-Gruppe tätig. Von 2005 bis 2008 war er Landesbeauftragter der Konrad-Adenauer-Stiftung in Bolivien und leitet seit September 2008 das Regionalprogramm „Medien und Demokratie in Lateinamerika" mit Sitz in Buenos Aires / Argentinien. Email: PeterAlberto.Behrens@kas.de

\title{
Zur Problematik des „Beratens“ und „beraten Werdens“
}

\section{Beratungsarbeit der GIZ in Malawi}

Jens Hiller

\section{Kernaussagen:}

Politikberatung kann an verschiedenen Faktoren scheitern. Diese Faktoren umfassen neben strukturellen und systemischen Merkmalen auch das persönliche Verhältnis zwischen Berater und Ratsuchenden. Verstärkt werden diese beratungshemmenden Faktoren zusätzlich, wenn ein sozio-politisches System beraten wird, welchen von den europäischen Normen abzuweichen scheint. Am Beispiel der Beratungsarbeit der GIZ im afrikanischen Staat Malawi wird gezeigt, dass vorab klare Zielsetzungen, Transparenz, Kooperation und Kompetenzverteilungen diesen Faktoren entgegenwirken können.

\section{Einleitung}

Es ist ein zentrales Thema für jeden Politikberater: Wann gelingt Politikberatung und wann gelingt sie nicht? Die Ant- worten auf diese Frage sind wohl so unterschiedlich wie die Antwortgeber selbst. Bisweilen eindeutige Ergebnisse in der Form eines Methodenkoffers, aus denen sich Politikberater bedienen können um sich eines Erfolgs ihrer Beratungsleistung sicher sein zu können, fehlen. Stattdessen scheint jeder Politikberater seine eigene Vorstellung zu haben, wann Politikberatung funktioniert und wann nicht. Das vielzitierte Konzept des Politikberaters als „romantischen Ironiker" (Heinelt, 2011, S. 54) stellt nur eine Variante dar. Dieser Bericht soll und kann indes diese Frage nicht beantworten. Stattdessen soll er gelungene und weniger gelungene Voraussetzungen für eine optimale Beratung aufzeigen.

Wer die Frage nach einem Gelingen von Politikberatung stellt, stellt automatisch die Frage nach dem Verhältnis zwischen Berater und dem zu Beratendem. Dieses Verhältnis ist fundamental für Politikberatung und vor allem Politikberater. Ist der Ratsuchende tatsächlich an einer rationalen und objektiven Beratung interessiert oder versucht er lediglich seine 
bereits gefällten Entscheidungen durch den Namen und die Expertise des Beraters zu rechtfertigen? Begegnen sich Berater und Ratsuchender auf Augenhöhe, oder existiert ein aufgezwungenes oder freiwillig eingenommenes Experten/Laien Verhältnis? Treffen die Ergebnisse der Beratung auf Zustimmung oder Ablehnung? Diese Fragen zeigen nur einen kleinen Teil der Problematik in der Wechselwirkung des „Beratens“ und des „sich beraten lassen“ auf.

\section{Zum grundlegenden Verhältnis zwischen Berater und Ratsuchenden}

Wie D. Messner in seiner Vorlesung zum Thema „Wissenschaftliche Politikberatung für globale nachhaltige Entwicklung“ (12. Mai 2012) äußerte, stehen Politikberater zunächst in einem spezifischen Dienstleistungsverhältnis zu dem Ratsuchenden. Dies ist das grundlegendste Arbeitsverhältnis zwischen beiden Seiten. Der Politikberater, unabhängig davon ob es sich hierbei um eine einzelne Person, ein Institut oder eine Stiftung handelt, bietet in erster Regel wissenschaftlich fundiertes und kommuniziertes Fachwissen an - ein Fachwissen, welches der Ratsuchende nachfragt. Aber eben dieses Fachwissen bürgt u.a. bereits ein nicht zu unterschätzendes Konfliktpotenzial für das Verhältnis zwischen Berater und zu Beratenden. Zum einen gibt Fachwissen nur selten eindeutige Vorgaben vor. Etwaiger Wunsch seitens der Ratsuchenden auf eine eindeutige best practise Lösung muss seitens der Berater meist zurückgewiesen werden. Zum anderen sind die theoretischen Erkenntnisse nur schwerlich auf die angetroffenen Zustände anzuwenden. Transferleistungen von der Theorie zur Praxis sind nötig. Gleichzeitig birgt der Umstand des fachwissenschaftlich fundierten Expertenwissens die Gefahr, dass der theoretisch gebildete Berater und der praxisorientierte Ratsuchende nicht dieselben Ziele und Vorstellungen verfolgen. Diese Problematik soll im Weiteren am Beispiel der Beratungsarbeit der Deutschen Gesellschaft für internationale Zusammenarbeit (GIZ) im afrikanischen Staat Malawi verdeutlicht werden.

\section{Politikberatung außerhalb Europas - Das Beispiel Malawi}

Seit 2010 berät die GIZ im Auftrag des Bundesministeriums für wirtschaftliche Zusammenarbeit (BMZ) die Regierung Malawis mit dem Ziel, die Grundbildung im Staat zu verbessern. Ziel der Beratung ist eine Binnenmodernisierung (vgl. Wewer, 2011, S. 106) des Bildungssektors sowie der daran angeschlossenen Teilsektoren. Dabei setzt die GIZ am Schnittpunkt zwischen second order governing und first order governing (vgl. Heinelt, 2011, S. 48 f) an. Die Beratungsleistung zielt sowohl auf institutionelle als auch auf organisatorische Ebene ab. Wie bei den meisten Beratungsprojekten soll auch hier die Funktionalität und die Effektivität der malawischen Grundbildung verbessert werden.

Soweit handelt es sich bei der Arbeit der GIZ in Malawi um eine klassische Politikberatung von staatlichen Institutionen, wie sie auch in Deutschland oder anderen europäischen
Staaten vorkommt. Allerdings stellt sich auf Grund der Exklusivität der malawischen Staatsstrukturen und der spezifischen sozio-ökonomischen Umstände eine Reihe von Besonderheiten für die Beratung in den Weg. Diese Besonderheiten stellen bestimmte Anforderungen an die Beratungsarbeit der GIZ. Diese sollen im Weiteren aufgezeigt werden.

Das 14 Millionen Einwohner Land gehört zu den ökonomisch rückständigsten Ländern der Welt. Der Human Development Index weist Malawi auf den 171. Platz aus (Stand 2012). Die HIV/AIDS Rate ist, wie in den meisten südafrikanischen Staaten, sehr hoch und bedeutet eine starke Fluktuation der Ansprechpartner in malawischen Ministerien. Transparency International listet Malawi in seinen Korruptionsindex mit 3 Punkten als sehr Korruptionsanfällig auf (Stand 2011). Mit einem Pro-Kopf Einkommen von ca. 260 Euro und einem Bevölkerungswachstum von ca. 3\% (GIZ 2012) sind zukunftsorientierte und nachhaltige Reformen in verschiedenen staatlichen Sektoren dringend notwendig. In diese Zustände eingebettet versucht nun die GIZ eine Beratungsleistung zu vollbringen, die auf die Modernisierung von staatlichen Strukturen abzielt. Dabei präsentiert sich die GIZ als eine Expertenorganisation. Die Mitarbeiter der GIZ sind in ihren Aufgaben geschult und verfügen sowohl über theoretisches Fachwissen als auch praktisches Transferwissen, welches von verschiedenen vorhergehenden Projekten weltweit erprobt wurde. Auf der anderen Seite sind die Offiziellen der malawischen Regierung und vor allem des Bildungsministeriums an einer Beratung der GIZ interessiert. Nichtsdestotrotz ergeben sich auf Grund der spezifischen Umstände im Land Problematiken, welche die Beratung und die Beratungsannahme erschweren.

\subsection{Konflikte durch ökonomische Faktoren}

Eine der weitreichendsten Problematiken im Beratungsprozess stellt die wirtschaftliche Lage des malawischen Staatshaushalts dar. Jede vorgeschlagene Veränderung oder Neuerung ist mit Kosten verbunden. Kosten, welche das malawische Haushaltsbudget nicht stemmen kann. Als Beispiel sei hier die Verbesserung der Grundbildung in ländlichen Gebieten angeführt. Um die Grundbildung einem möglichst großen Teil der Bevölkerung zukommen zu lassen sind Umstrukturierungsprozesse des Bildungssystems und der Bildungsstruktur von Nöten. Hier entsteht ein erster Konfliktpunkt zwischen dem Wechselverhältnis des „beraten Wollens“ und „sich beraten lassen“. Für die GIZ ist eine Verbesserung der Grundbildung mit einer weitreichenden Dezentralisierung des Verwaltungsapparats, Reformen der Bildungspläne und einer Verbesserung der Lehrerbildung (GIZ 2012) verbunden. Für die Berater der GIZ steht der Sachgegenstand im Mittelpunkt. Es kommt zu einer Fokussierung auf die Fragestellung, wie die Grundbildung verbessert werden könnte. Diese Fokussierung auf ein isoliertes Thema ist hierbei nicht negativ auszulegen, erfüllt es doch genau die Aufgabe, für welche der Berater engagiert wurde. Verbesserungsmöglichkeiten werden dann auf Grundlage des Fachwissens getroffen und dem Ratsuchen- 
den vorgelegt. Obwohl der Ratsuchende, hier die malawische Regierung bzw. das malawische Bildungsministerium, die Notwendigkeit dieser Vorschläge auch im Hinblick der oben angeführten Lage im Land akzeptiert, wurden und werden etwaige Reformen zum Teil nur zögerlich umgesetzt. Der Grund ist, dass der Ratsuchende neben der Zentrierung auf den Sachgegenstand, weiterführende und mit anderen Teilbereichen verknüpfte Wechselwirkungen vor Augen hat. In diesem Fall bedeutet dies, dass ca. 30\% des malawischen Bildungsetats durch Geberländer finanziert werden. Bei einer solch hohen Abhängigkeit von anderen politischen Akteuren, denen Rechenschaft abgelegt werden muss, um die Hilfen verlängert zu bekommen, steht der Schwerpunkt der Betrachtung des Sachgegenstandes verständlicherweise nicht mehr auf der Verbesserung der Effektivität sondern auf dem bloßen Erhalt. Anders ausgedrückt ist für den Ratsuchenden die Aufrechterhaltung der Unterstützung durch Dritte wichtiger als eine Optimierung im Politics Bereich. Insbesondere wenn etwaige Reformen mit zusätzlichen Kosten verbunden sind, welche dann womöglich bei Dritten eingefordert werden müssten. Somit birgt dieser Umstand die Gefahr, dass vom Berater vorgelegte Entscheidungsmöglichkeiten vom Ratsuchenden gezielt geblockt oder hinausgezögert werden, da sie entweder keine Priorität oder ein gegenläufiges Interesse darstellen. Eine Beratung vor diesem Hintergrund gestaltet sich als schwierig, sind doch die Zielsetzungen in einem unterschiedlichen Maße gewichtet. Somit kommt es zu einen Form der Pattsituation. Der Berater gibt die Entscheidungsmöglichkeiten vor, welche zu einer Optimierung des Sachgegenstandes führen sollen. Der Ratsuchende, wissend ob des notwendigen Reformdrucks, ist jedoch durch seine starke finanzielle Abhängigkeit in einem Zerreiszustand. Optimierungsmöglichkeiten werden aufgeschoben, verworfen oder nur langsam umgesetzt.

\subsection{Konflikte durch unzureichende Kooperation}

Allerdings können nicht nur verschiedenartige Prioritäten ein Problem für das Verhältnis zwischen Berater und zu Beratenden werden, sondern ebenfalls der Grad und die Qualität der Kooperation. Intransparente Verwaltungsprozesse, mangelnde Kompetenzklärungen oder unzureichende organisatorische Klarheit behindern das Verhältnis zwischen Berater und zu Beratenden. Staatliche Institutionen neigen zu eigenen, für Dritte meist schwer zu durchschauenden Entscheidungs- und Umsetzungsprozessen. Jedoch ist es gerade für Politikberater unumgänglich, dass eben jene Strukturen erkannt, durchschaut und in Umstrukturierungsprozesse miteingeplant werden. Beratungsaufgaben, welche auf die Optimierung von institutionellen Prozessen abzielen, können dies nur auf der Grundlage der der Optimierung gegenläufigen Zustände tun. Nichtdestotrotz benötigen Berater während ihrer Arbeit Informationen, Ansprechpartner und vor allem eine gesunde Kooperation, um ihre Aufgabe gerecht zu werden. Sind diese Voraussetzungen nicht geschaffen, ziehen sich Beratungsarbeiten unnötig in die Länge und verkomplizieren die Arbeit.
Diese undurchsichtigen staatlichen Strukturen finden sich auch in Malawi wieder. Die Zuständigkeit der Ansprechpartner der GIZ im malawischen Bildungsministerium ist zumeist ungeklärt und wechselhaft. Seitens der malawischen Ansprechpartner wird nur eine geringe persönliche Verantwortung für die Belange der Berater übernommen. Zuständigkeiten werden nach dem Prinzip des Erfolgs und des Interesses übernommen. Bring ein Projekt einen gewissen Erfolg oder ein gewisses Prestige, so wird die Verantwortung und Zuständigkeit in der Regel augenblicklich übernommen, misslingt ein Projekt oder bringt es nicht die gewünschten Resultate, so wird jegliche Verantwortung und Zuständigkeit abgelehnt. Auch die mangelnde öffentliche Repräsentanz der malawischen Staatsbeamten verkompliziert das Verhältnis zwischen Berater und zu Beratenden. Die malawischen Ministerialbeamten verfügen beispielsweise über keine offiziellen Kommunikationskanäle. Etwaige schriftliche Kommunikation findet über die privaten Mailadressen der Mitarbeiter statt. Dies macht die Kommunikationswege länger und weniger spezifisch. Rückantworten oder die Weitergabe von Memos oder Informationen verzögern sich auf diese Weise. Dies macht die Kooperation zwischen Berater und zu Beratenden äußerst schwierig. Eine nachhaltige Kooperationsbasis mit festen Ansprechpartnern und geklärten Verantwortlichkeiten ist auf dieser Grundlage nicht zu erreichen. Resultat dieser Umstände ist eine gewisse Unsicherheit seitens der Berater und eine Verkomplizierung der Beratungsleistung. Andererseits bringt auf Grund dieser Verzögerungen die Beratungsleistung nicht den gewünschten Erfolg seitens der Ratsuchenden.

\subsection{Konflikte durch Schattenwirtschaft}

Stellen die oben beschriebenen Problempunkte im Verhältnis zwischen Berater und zu Beratenden allgemein gültige Punkte dar, welche in dieser Form ebenfalls auch in Deutschland oder anderen europäischen Ländern vorkommen können, so gibt es ebenfalls auch spezifische Diskrepanzmerkmale, mit welchen Berater im Besonderen in nicht-westeuropäischen Staaten konfrontiert werden können. Hauptaugenmerk liegt hierbei in der vorherrschenden Korruption. Wird in Europa Korruption weitgehend als „Grauzonenphänomen“ (Priddat, 2009, S. 155) gesehen, ist sie in anderen Regionen zu einer konstanten Größe avanciert. Korruption tritt vor allem dort auf, wo finanzielle Anreize und persönliches Streben aufeinander treffen. Vor allem in Staaten, wo Staatsangestellte über ein geringes Grundeinkommen verfügen und staatliche Kontrollen weitgehend inexistent sind, findet sich eine erhöhte Korruptionsanfälligkeit vor. Getreu dem Motto: „Wer [...] nicht besticht, bekommt normale Leistungen [...] wer extra zablt, [...] bekommt exquisite und schnelle Leistungen" (Ebd. S, 165, Hervorhebung im Original), ermatten staatliche Leistungen ohne Sonderzuzahlungen. Politikberatung, welche auf Verbesserung abzielt, trifft in diesem Kontext auch auf Ablehnung. Jegliche Veränderung der Gegebenheiten bedeutet eine Gefahr für etablierte irreguläre Transaktionsmuster. Der Bera- 
ter tritt hier als direkter Gegenspieler zur Korruption auf. Dies bedeutet zweierlei. Zum einen, dass der Berater verständlicherweise keine sonderliche Resonanz von seinem Gegenüber zu erwarten hat und zum anderen, dass der Berater seine Verbesserungsvorschläge gegen ein lukratives und bereits etablierten System durchsetzen muss. Eine Belastung sowohl für Berater als auch für zu Beratenden.

\section{Fazit}

Zusammenfassend kann am Beispiel der Beratungsarbeit der GIZ in Malawi eine Reihe von beratungshemmenden Faktoren aufgezeigt werden. Diese Faktoren beziehen sich allesamt auf das Verhältnis zwischen Berater und zu Beratenden. Unabhängig, ob es sich bei Politikberatung um Unternehmensberatung, Prozessberatung oder systemische Organisationsberatung (vgl. Simon, 2011, S. 261ff) handelt, steht immer die Wechselbeziehung zwischen beiden Parteien im Zentrum. Um etwaige Missverständnisse, unterschiedliche Prioritätssetzungen oder konträre Ziele zu vermeiden, empfiehlt es sich zu Beginn der Beratung gemeinsam eindeutige Zielsetzungen zu formulieren. Die Beteiligung von Dritten - nicht unüblich innerhalb der Politikberatung - kann und muss in den Beratungsprozess eingeplant werden, um eine optimale Zusammenarbeit zu gewährleisten. Auch die zumeist verwirrenden Prozessstrukturen innerhalb staatlicher Institutionen können durch die vorherige Klärung von Verantwortlichkeit und Zuständigkeit bereinigt werden. Der Berater, der wie oben bereits erwähnt nach Messner eine Dienstleistung erbringt, muss sich ebenfalls neben dem eigentlichen Sachgegenstand auf verschieden „Nebengebiete“ einstellen (vgl. Lieber, 2011, S. 167). Politikberater finden selten optimale Zustände vor. Wäre es so, dann würde ihre Beratung überflüssig werden. Allerdings zeigen die oben erwähnten Beispiele, dass das Gelingen von Beratung nicht nur im Ermessen des Beraters liegt, sondern zu gleichen Teilen auch auf der anderen Seite Gehör finden muss. Wer Beratung anfordert, weil er selbst von innen heraus nicht in der Lage ist, optionale Wege zu erkennen und einzuschlagen, muss auch offen und Willens für Beratungsvorschläge sein. Das Kooperationsverhältnis zwischen Berater und zu Beratenden sollte daher auf einer beidseitigen freundschaftlichen und ehrlichen Basis beruhen.

Abschließend sei hier noch erwähnt, dass trotz dieser in diesem Bericht angeführten beratungshemmenden Faktoren,
Politikberatung auch langwierige Erfolge verbuchen kann. Durch die Beratung und Unterstützung der GIZ konnte die Lehrerbildung und die Zahl der Schulabschlüsse in Malawi qualitativ verbessert werden. Durch diese Verbesserungen wurde der malawischen Regierung erfolgreich Mittel aus der Global Partnership for Education - Initiative bewilligt (GIZ 2012).

\section{Literatur}

Deutsche Gesellschaft für internationale Zusammenarbeit (2012): Malawi. Verfügbar unter http://www.giz.de/de/weltweit/341.html [28.9.2012]

Heinelt, H. (2011): Ratsuchende Politiker, beratende Wissenschaftler und verschiedene Ebenen politischen Handelns. In: Lamping, W. \& Schridde, H. (Hrsg.): Der konsultative Staat. Opladen: Budrich

Human Development Index (2012): Country Profile Malawi. Verfügbar unter http://hdrstats.undp.org/en/countries/profiles/MWI.html [28.9.2012]

Lamping, W. \& Schridde, H. (2011): Wer ist Koch und Wer ist Kellner? In: Lamping, W. \& Schridde, H. (Hrsg.): Der konsultative Staat. Opladen: Budrich.

Lieber, A. (2011): Deutsche wirtschaftspolitische Reformberatung in China am Beispiel der GTZ. In: Zeitschrift für Politikberatung, Heft 4. S. 164-167.

Priddat, B. P. (2009): Politik unter Einfluss. Netzwerke, Öffentlichkeiten, Beratungen, Lobby. Wiesbaden: VS

Simon, F. B. (2011): Praktisch funktioniert es, aber tut es das auch theoretisch? System-theoretisch-konstruktivistische Perspektive der Politikberatung. In: Lamping, W. \& Schridde, H. (Hrsg.): Der konsultative Staat. Opladen: Budrich.

Transparency International (2011): Corruption Perceptions Index 2011. Verfügbar unter http://cpi.transparency.org/cpi2011/results/ [28.9.2012] Wewer, G. (2011): Staatsmodernisierung und Politikberatung. In: Lamping, W. \& Schridde, H. (Hrsg.): Der konsultative Staat. Opladen: Budrich

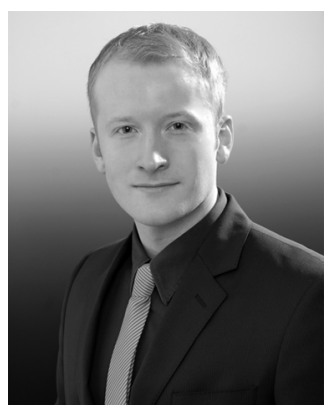

Jens Hiller, geb. 1986, ist wissenschaftlicher Mitarbeiter für Soziologie und Lehrbeauftragter für Politikwissenschaft an der Bergischen Universität Wuppertal. Er forscht und lehrt im Bereich der Konfliktforschung mit Schwerpunkten auf Konflikte mit niedriger Intensität und Diskursanalysen. 